01,13

\title{
Уменьшение обменного смещения, вызванное ростом эффективной толщины слоя меди в гетероструктурах $\mathrm{NiFe} / \mathrm{Cu} / \mathrm{IrMn}$
}

\author{
(C) Р.Б. Моргунов, М.В. Бахметьев, А.Д. Таланцев \\ Институт проблем химической фризики РАН, \\ Черноголовка, Россия \\ E-mail: morgunov2005@yandex.ru
}

Поступила в Редакцию 19 июня 2020 г.

В окончательной редакции 19 июня 2020 г.

Принята к публикации 9 июля 2020 г.

B серии структур $\mathrm{NiFe} / \mathrm{Cu} / \mathrm{IrMn}$ с варьируемой эффективной толщиной немагнитной прослойки меди (вплоть до ее отсутствия в образце $\mathrm{NiFe} / \mathrm{IrMn}$ ) наблюдается уменьшение обменного смещения и коэрцитивной силы при увеличении эффективной толщины слоя меди. Слияние островков меди при достижении эффективной толщины слоя $\mathrm{Cu} 1 \mathrm{~nm}$ вызывает смену контактного обменного взаимодействия между ферро- и антиферромагнетиком $\mathrm{NiFe}-\mathrm{IrMn}$ на косвенное обменное взаимодействие через электроны проводимости слоя меди $\mathrm{NiFe}-\mathrm{Cu}-\mathrm{IrMn}$. При этом структурное качество монокристаллических феррои антиферромагнитных слоев не изменяется, а перемагничивание осуществляется без участия доменов в диапазоне температур 2-300 К. Моделирование динамики напыления демонстрирует островковую структуру пленки на начальных стадиях до эффективной толщины $1 \mathrm{~nm}$.

Ключевые слова: гетероструктура, ферромагнетик-антиферромагнетик, обменное смещение, обменное взаимодействие.

DOI: 10.21883/FTT.2020.11.50050.133

\section{1. Введение}

Гетероструктуры, содержащие ферромагнитный (FM) слой и антиферромагнитный (AFM) слой, широко используются в качестве активных элементов датчиков Холла [1] и устройств магнитной памяти [2] потому, что они проявляют эффект обменного смещения, устанавливающий интервал полей, в котором используются эти устройства. Обменная связь позволяет закрепить направление намагниченности части слоя FM вблизи интерфейса FM/AFM [3]. Обменное взаимодействие между ферро- и антиферромагнетиком приводит к сдвигу петли гистерезиса вдоль оси поля и создает условия для постоянной стабильной и всегда одинаковой намагниченности гетероструктуры в исходном состоянии в нулевом магнитном поле. Это делает устройства энергонезависимыми, поскольку „записанная“ однажды намагниченность требует значительно большего поля для размагничивания структуры. Кроме того, устройство становится независимым от числа и стадии завершения измерительных циклов [4]. Сочетание намагничивания области AFM вблизи интерфейса слоя FM с антиферромагнитным обменом на интерфейсе FM/AFM приводит к смещению центра петли гистерезиса в отрицательные поля [5]. Смещение петли гистерезиса зависит от совершенства интерфейса FM/AFM [6], толщины ферромагнитного [7] и антиферромагнитного слоев [8], кристалличности этих слоев [9] и от используемых материалов ферро- и антиферромагнетика [10]. В настоящее время изготавливают магнитные структуры с обменной связью, в которых обменное смещение превышает коэрцитивную силу [11]. Среди большого разнообразия систем с обменной связью, магнитная структура $\mathrm{NiFe} / \mathrm{IrMn}$ имеет самое большое соотношение между обменным смещением и коэрцитивной силой [12].

Для увеличения чувствительности датчиков, в магнитные структуры FM/AFM вводят немагнитную прослойку (NM) между ферромагнитным и антиферромагнитным слоями [13]. Это приводит к регулируемому уменьшению обменного смещения [13-16] и отчасти решает задачу настройки соответствующего рабочего интервала магнитных полей. Этот подход основан на том, что тонкая немагнитная прослойка (в несколько атомных слоев) не вызывает существенных изменений в распределении электрического тока в гетероструктуре $\mathrm{NiFe} / \mathrm{IrMn}$. Поэтому общая проводимость магнитной структуры $\mathrm{NiFe} / \mathrm{NM} / \mathrm{IrMn}$ и ее магнитосопротивление остаются такими же, как и в структуре $\mathrm{NiFe} / \mathrm{IrMn}$, позволяя управлять обменным смещением без изменения эффективности переключения прибора полем [13].

Требование небольшой толщины немагнитной прослойки вызывает ряд проблем с повторяемостью ее воспроизведения, с качеством напыления и предсказуемостью полученного результата. Зависимость обменного смещения от эффективной толщины немагнитной прослойки $t_{\mathrm{NM}}$, определяемой по времени напыления слоя NM, в системах FM/NM/IrMn различна в экспериментах разных групп и противоречива в интерпретации. В [14], в структурах $\mathrm{CoFe} / \mathrm{NM} / \mathrm{IrMn}(\mathrm{NM}=\mathrm{Ag}$, $\mathrm{Au}, \mathrm{Si}, \mathrm{Pd}, \mathrm{Ru})$ наблюдалась экспоненциальная зависимость $H_{b} \sim \exp \left(-t_{\mathrm{NM}} / t_{0}\right)$, логично воспринимаемая, как следствие экспоненциального затухания спиновой поляризации электронов в немагнитном металле. Однако 
прослойка титана давала немонотонную зависимость. В [15] зависимость $H_{b}\left(t_{\mathrm{NM}}\right)$ содержала ступеньку и была описана с помощью Гауссова распределения обменного смещения, ширина которого менялась с ростом $t_{\mathrm{NM}}$ в структурах $\mathrm{IrMn} / \mathrm{Cu} / \mathrm{Co}$. В [16] наблюдалась немонотонная зависимость $H_{b}\left(t_{\mathrm{NM}}\right)$ с максимумом в структурах $\mathrm{Pt} / \mathrm{Co} / \mathrm{Pt} / \mathrm{IrMn}$, которая объяснялась вкладом слоя платины в перпендикулярную анизотропию. Для систем, где плоскость интерфейса однородно покрыта лишь одним атомным слоем NM, обменное смещение при низких температурах существует, но при комнатной температуре полностью исчезает [17]. Изучались температурные зависимости обменного смещения при различных толщинах немагнитной прослойки [18], но эти данные были получены лишь для узкого диапазона толщин немагнитной прослойки, который не охватывал все ситуации от полностью непокрытого до полностью покрытого слоя NM. Наконец, известно, что наличие немагнитного слоя способно влиять на рост, качество и текстурирование ферромагнитного и антиферромагнитного слоев, радикально изменяя свойства готового прибора [19].

Целью настоящей работы является анализ обменного взаимодействия гетероструктур $\mathrm{NiFe} / \mathrm{Cu} / \mathrm{IrMn}$ и исследование поведения обменного смещения и коэрцитивной силы в них, в таком интервале эффективной толщины немагнитной прослойки $\mathrm{Cu}$, который охватывает все типы покрытия поверхности ферромагнетика медью.

\section{2. Методика}

Гетероструктуры были напылены на подложках $\mathrm{Si} / \mathrm{SiO}_{2}$ методом магнетронного напыления на постоянном токе (DC magnetron sputtering), подробно методика изготовления структур описана в работе [20]. В опытах использовали двухслойный образец $\mathrm{Ta}(5 \mathrm{~nm}) / \mathrm{NiFe}(10 \mathrm{~nm}) / \operatorname{IrMn}(10 \mathrm{~nm}) / \mathrm{Ta}(5 \mathrm{~nm})$ и серию трехслойных образцов $\mathrm{Ta}(5 \mathrm{~nm}) / \mathrm{NiFe}(10 \mathrm{~nm}) / \mathrm{Cu}(\mathrm{tCu}) /$ $\operatorname{IrMn}(10 \mathrm{~nm}) / \mathrm{Ta}(5 \mathrm{~nm})$ (рис. 1), в которых эффективная толщина немагнитной прослойки $t_{\mathrm{Cu}}$, определяемая по длительности напыления, варьируется в диапазоне от 0.08 до $5 \mathrm{~nm}$. Нижняя граница этого диапазона соответствует такому количеству атомов меди, которое даже при идеально ровном распылении в один слой не покроет всю поверхность образца. Верхняя граница диапазона отвечает такому количеству меди, при котором поверхность будет покрыта полностью слоем меди, а произвольные вариации ее толщины будут значительно меньше самой толщины $t_{\mathrm{Cu}}$. С целью буферизации дефектов и усиления текстуры (111) слоев NiFe и IrMn, перед слоем $\mathrm{NiFe}$ напылялся слой Та, что позволяло получать структуры с величиной отношения поля обменного смещения к коэрцитивной силе большей, чем в случае аналогичных структурах без буфера. Так как в образцах с толщиной немагнитной прослойки $t_{\mathrm{Cu}}<1 \mathrm{~nm}$, эффективная толщина прослойки $\mathrm{Cu}$ недостаточна для полного покрытия интерфейса даже в случае однородного

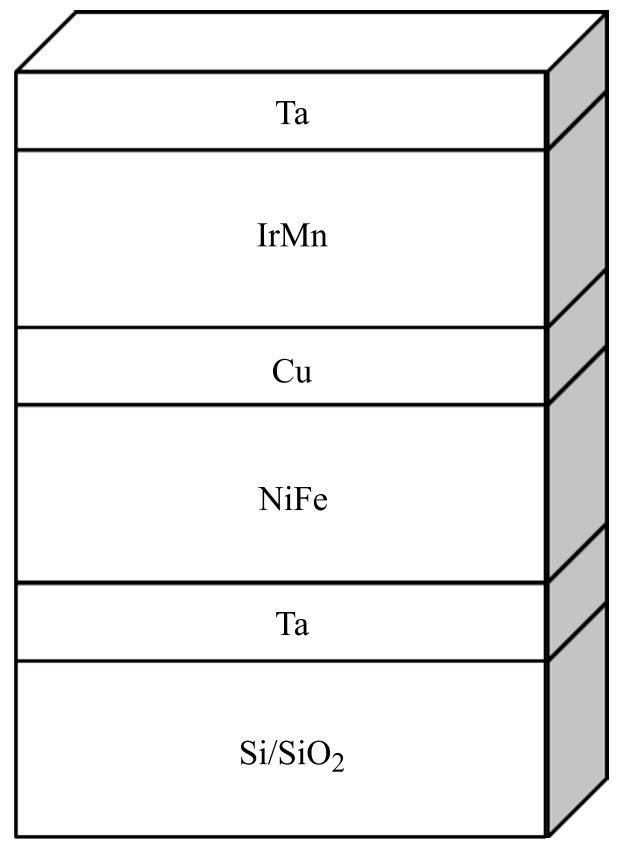

Рис. 1. Схема гетероструктур двухслойного образца $\mathrm{Ta}(5 \mathrm{~nm}) / \mathrm{NiFe}(10 \mathrm{~nm}) / \mathrm{IrMn}(10 \mathrm{~nm}) / \mathrm{Ta}(5 \mathrm{~nm})$ и трехслойных образцов $\mathrm{Ta}(5 \mathrm{~nm}) / \mathrm{NiFe}(10 \mathrm{~nm}) / \mathrm{Cu}(\mathrm{tCu}) / \mathrm{IrMn}(10 \mathrm{~nm}) / \mathrm{Ta}(5 \mathrm{~nm})$.

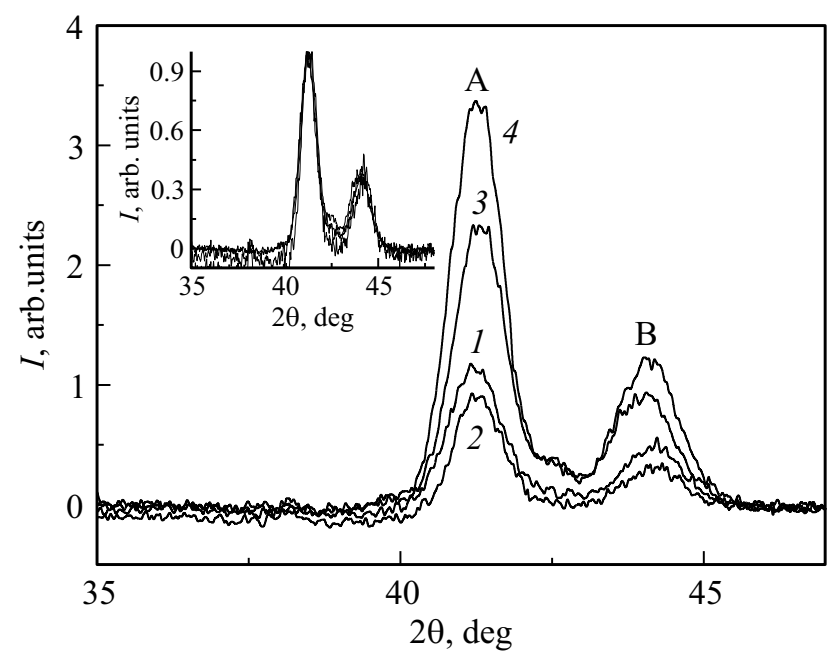

Рис. 2. Рентгеновские дифракционные спектры образцов с эффективными толщинами слоя Сu 0 (1), 0.08 (2), 1.04 (3), $2.5 \mathrm{~nm}$ (4). Линия А соответствует $\operatorname{IrMn}(111)$, а линия В $\mathrm{Ni}_{0.8} \mathrm{Fe}_{0.2}$ (111). На врезке нормированные спектры этих же образцов.

распределения атомов, немагнитная прослойка распределяется островками. При эффективной толщине немагнитной прослойки $t_{\mathrm{Cu}}>1 \mathrm{~nm}$ атомов меди становится достаточно для полного покрытия интерфейса. Это не гарантирует a priori, что полное покрытие реализуется на самом деле.

Данные рентгенографии были получены с помощью дифрактометра ДРОН-3М с использованием медной трубки $\left(K_{\alpha_{1}}\right.$-рентгеновские лучи с длиной волны $\left.1.54 \AA\right)$, 

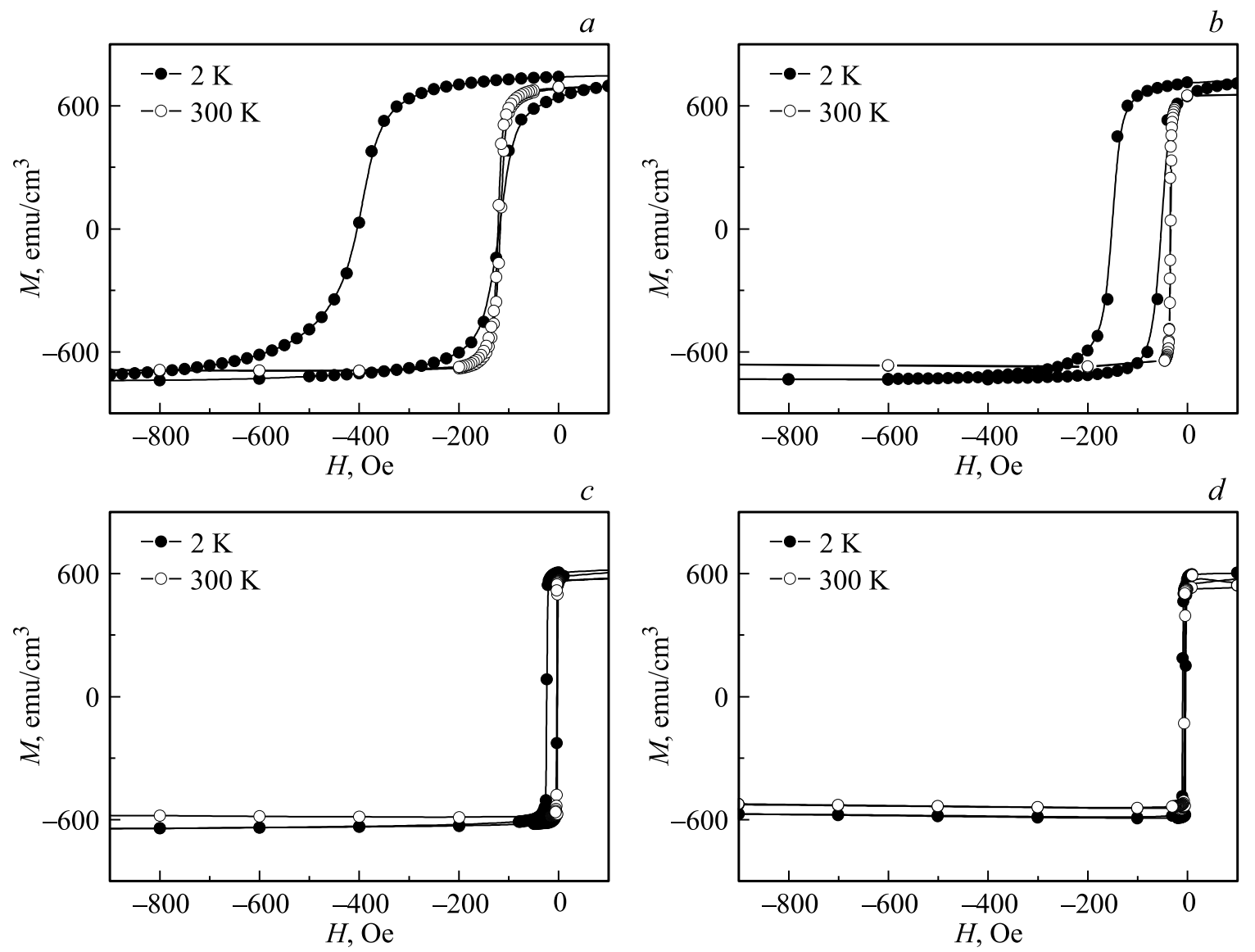

Рис. 3. Петли гистерезиса образцов с эффективными толщинами слоя $\mathrm{Cu} 0(a), 0.08(b), 1.04(c), 2.5 \mathrm{~nm}(d)$ при температурах 2 и $300 \mathrm{~K}$.

работающей при $35 \mathrm{kV}$ и $25 \mathrm{~mA}$ со стабильностью $0.01 \% / 8 \mathrm{~h}$. Измерения каждой системы были выполнены в диапазоне углов $2 \theta=30-50^{\circ}$ с шагом $0.2^{\circ}$. Спектры рентгеновской дифракции для образцов $\mathrm{Cu} 0 \mathrm{~nm}(1)$, $\mathrm{Cu} 0.08 \mathrm{~nm}$ (2), Cu $1.04 \mathrm{~nm}(3), \mathrm{Cu} 2.5 \mathrm{~nm}$ (4), показаны на рис. 2. Пики IrMn соответствуют $2 \theta=41.3^{\circ}$ - линия $\mathrm{A}$, пики $\mathrm{NiFe}$ соответствуют $2 \theta=44.2^{\circ}$ - линия $\mathrm{B}$, на врезке представлены нормированные спектры этих же образцов. Слои IrMn и NiFe имеют кубическую (111) структуру [21]. Неизменность спектра рентгеновской дифракции при напылении слоев $\mathrm{Cu}$ любой толщины из исследуемого диапазона говорит о том, что прослойка меди не влияет на кристаллическое качество слоев IrMn и NiFe.

Измерения зависимостей магнитного момента от магнитного поля и от температуры, были проведены на SQUID-магнетометре MPMS 5XL Quantum Design на образцах размерами $5 \times 5 \mathrm{~mm}^{2}$, в магнитных полях $H=-2 \mathrm{kOe}-2 \mathrm{kOе}$ и при температурах $T=2-300 \mathrm{~K}$. Диамагнитный вклад подложки был вычтен.

\section{3. Результаты и обсуждение}

На рис. 3, а показаны петли гистерезиса двухслойного образца без немагнитной прослойки $\mathrm{Cu}$. Видно, что при температуре $2 \mathrm{~K}$, поле обменного смещения $H_{b}$ и коэрцитивная сила $H_{c}$ имеют наибольшие значения $\left(H_{b}=261 \mathrm{Oe} ; H_{c}=142 \mathrm{Oe}\right)$. С повышением температуры $H_{b}$ и $H_{c}$ становятся меньше (например, при температуре $\left.300 \mathrm{~K} H_{b}=120 \mathrm{Oe} ; H_{c}=2.4 \mathrm{Oe}\right)$. С увеличением эффективной толщины немагнитной прослойки $\mathrm{Cu}$ в диапазоне толщин от 0 до $5 \mathrm{~nm}$, поле обменного смещения и коэрцитивная сила уменьшаются (рис. $3, b, c, d)$.

Температурные зависимости поля обменного смещения $H_{b}$ и коэрцитивной силы $H_{c}$ показаны на рис. 4, $a$ и $b$, для образцов $\mathrm{Cu} 0, \mathrm{Cu} 0.5, \mathrm{Cu} 1.04 \mathrm{~nm}$. Видно, что высокотемпературная часть зависимостей $H_{b}(T)$ (в диапазоне температур 50-300 K) близка к линейным, в то время как низкотемпературная часть зависимостей $H_{b}(T)(2-50 \mathrm{~K})$, претерпевает резкий рост с понижением температуры. Высокотемпературную часть температурных зависимостей $H_{b}(T)$ можно отнести к прямому обмену между $\mathrm{NiFe}$ и $\mathrm{IrMn}$, а низкотемпературную часть можно отнести к обмену через электроны проводимости $\mathrm{Cu}$ между $\mathrm{NiFe} / \mathrm{Cu} / \mathrm{IrMn}$ (косвенный обмен, исчезающий при высоких температурах). Чтобы разделить вклады обменных взаимодействий в фрагментах $\mathrm{NiFe}-\mathrm{IrMn}$, и в фрагментах $\mathrm{NiFe}-\mathrm{Cu}-\mathrm{IrMn}$, температурные зависимости поля обменного смещения были аппроксимированы суммой двух экспоненциальных зависимостей, учитыва- 

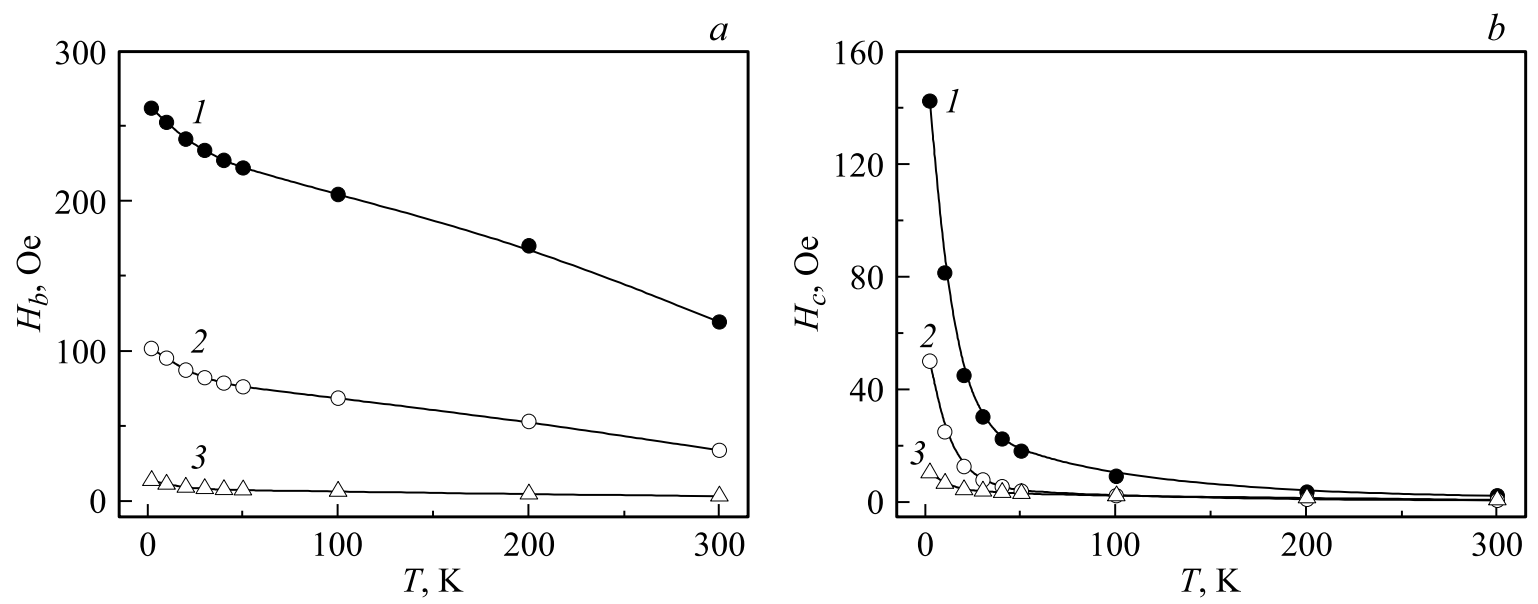

Рис. 4. Температурная зависимость поля обменного смещения $H_{b}$ образцов $\mathrm{Cu} 0$ (1), 0.5 (2), $1.04 \mathrm{~nm}(3)(a)$. Температурная зависимость коэрцитивной силы $H_{c}$ образцов $\mathrm{Cu} 0$ (1), 0.5 (2), $1.04 \mathrm{~nm}(3)(b)$.
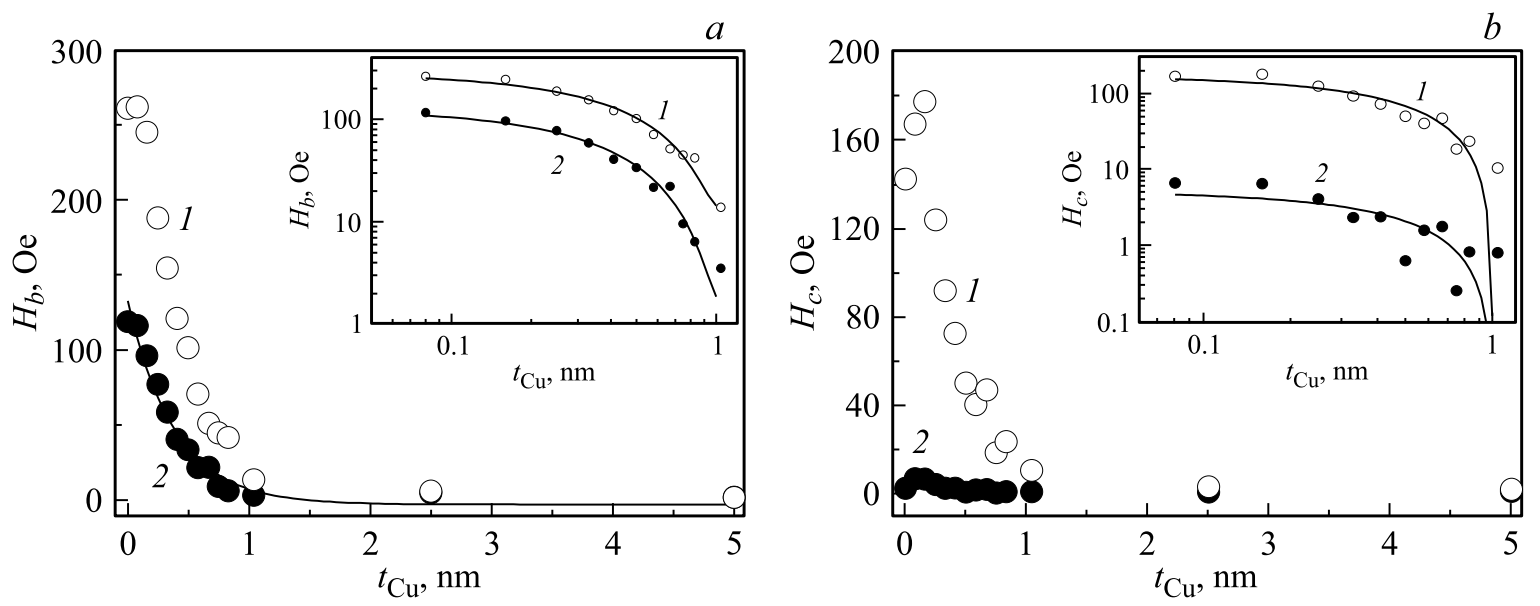

Рис. 5. Зависимость поля обменного смещения от эффективной толщины немагнитной прослойки $\mathrm{Cu}$, при температурах $T=2(1)$ и $T=300 \mathrm{~K}(2)(a)$, линией показана аппроксимация зависимости $H_{b}\left(t_{\mathrm{Cu}}\right)$ функцией $(2)$. Зависимость коэрцитивной силы от эффективной толщины немагнитной прослойки $\mathrm{Cu}$, при температуре $T=2(1)$ и $T=300 \mathrm{~K}(2)(b)$. На врезках показаны эти же зависимости в двойных логарифмических координатах.

ющих вклады каждого из типов фрагментов

$$
H_{b}(T)=A_{1} \exp \left(-\frac{T}{E_{1}}\right)+A_{2} \exp \left(-\frac{T}{E_{2}}\right) .
$$

Коэффициент $A_{1}$ - средняя величина обменного смещения в фрагментах $\mathrm{NiFe}-\mathrm{IrMn}, A_{2}$ - средняя величина обменного смещения в фрагментах $\mathrm{NiFe} / \mathrm{Cu} / \mathrm{IrMn}$ при условно нулевой температуре. Энергии $E_{1}$ и $E_{2}$ характеризуют соответствующие обменные взаимодействия в двух типах фрагментов. На рис. 4, $a$ показана аппроксимация температурных зависимостей обменного смещения формулой (1). Видно, что в рамках предложенного подхода удовлетворительно описана температурная зависимость этой величины.

Обменное взаимодействие через немагнитный слой меди в фрагментах $\mathrm{NiFe} / \mathrm{Cu} / \mathrm{IrMn}$ объясняется спинполяризованными электронами, способными передавать спиновую поляризацию через немагнитный металл. Такое обменное взаимодействие описывается в рамках теории Рудермана-Касуи-Киттеля-Йошиды (РККИ) [22]. Использование тяжелого металла Та с незавершенной $f$-оболочкой с высоким значением орбитального момента в качестве материала немагнитной прослойки [22] усиливает РККИ обмен между слоями $\mathrm{NiFe}$ и $\mathrm{IrMn}$ за счет увеличенной энергии спин-орбитального взаимодействия. Использование меди в данной работе приводит к тому, что обменное взаимодействие между слоями $\mathrm{NiFe}$ и IrMn и, соответственно, обменное смещение оказывается меньше по сравнению с [22] при прочих равных условиях.

Зависимости поля обменного смещения и коэрцитивной силы от эффективной толщины немагнитной прослойки $\mathrm{Cu}$ при температурах 2 и $300 \mathrm{~K}$ показаны на рис. $5, a, b$ соответственно. Поле обменного смещения с увеличением эффективной толщины немагнитной про- 

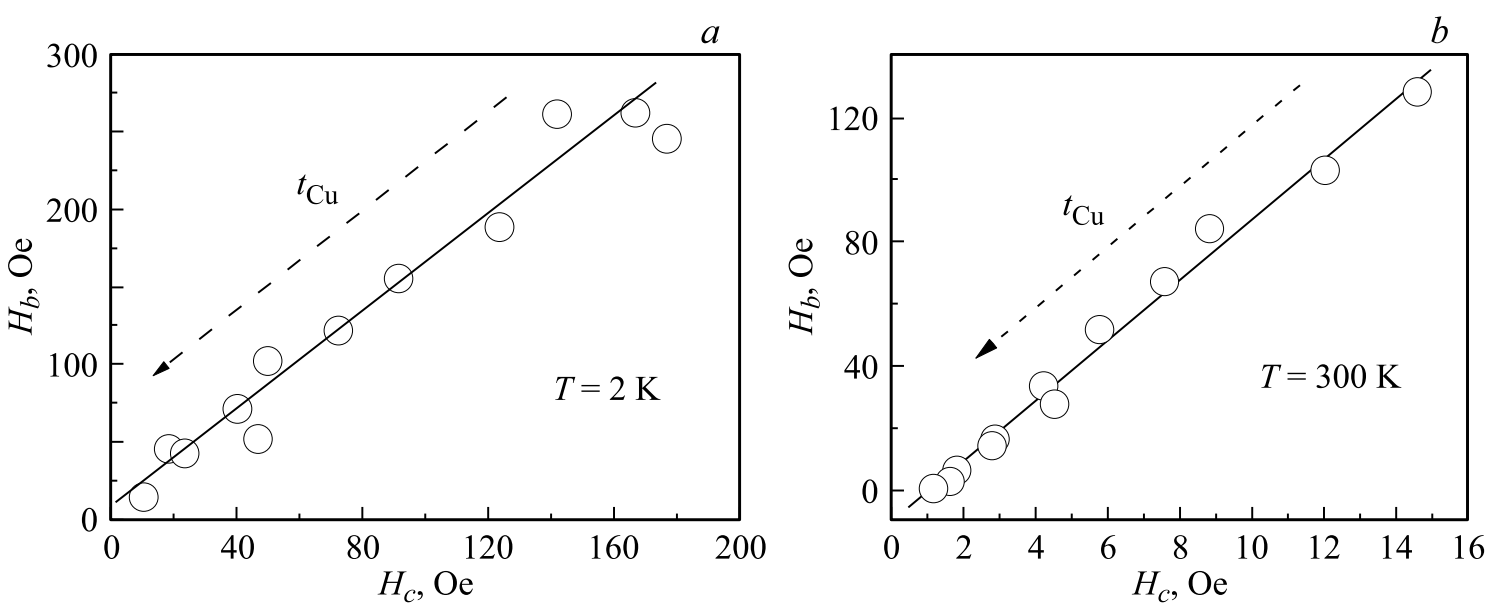

Рис. 6. Зависимость поля обменного смещения $H_{b}$ от коэрцитивной силы $H_{c}$ для температуры $T=2 \mathrm{~K}(a)$ и температуры $T=300 \mathrm{~K}(b)$.

слойки $\mathrm{Cu}$ убывает. При малых толщинах есть небольшое плато. Коэрцитивная сила с ростом эффективной толщины немагнитной прослойки $\mathrm{Cu}$, при комнатной температуре никак не изменяется и лежит в нулевых полях, но при температуре $2 \mathrm{~K}$, коэрцитивная сила сначала возрастает до $t_{\mathrm{Cu}}=0.16 \mathrm{~nm}$, затем убывает.

Экспериментальная зависимость поля обменного смещения от эффективной толщины немагнитной прослойки $\mathrm{Cu}$ (рис. 5, $a$ ), извлеченная с помощью формулы (1) из температурных зависимостей обменного смещения гетероструктур с разной толщиной слоя меди, может быть аппроксимирована экспоненциальной функцией

$$
H_{b}\left(t_{\mathrm{Cu}}\right)=H_{b 0} \exp \left(t_{\mathrm{Cu}} / \lambda_{\mathrm{Cu}}\right),
$$

где $H_{b 0}=120 \mathrm{Oе}$ - поле обменного смещения двухслойного образца $\mathrm{NiFe} / \mathrm{IrMn} ; \lambda_{\mathrm{Cu}}$ - длина затухания обменного смещения. Аппроксимация зависимости $H_{b}\left(t_{\mathrm{Cu}}\right)$ функцией (2) (сплошная линия на рис. 5, $a$ ) дает длину затухания $\lambda_{\mathrm{Cu}}=0.4 \mathrm{~nm}$. Это соответствует длине затухания РККИ взаимодействия и согласуется с независимыми данными в работе [14].

На рис. 6 показаны зависимости поля обменного смещения $H_{b}$ от коэрцитивной силы $H_{c}$ в условиях меняющейся толщины прослойки меди для температуры $T=2 \mathrm{~K}$ (рис. $6, a)$ и температуры $T=300 \mathrm{~K}$ (рис. $6, b)$. Направление изменения толщины прослойки показано стрелками. Из этих зависимостей следует, что с изменением эффективной толщины немагнитной прослойки $\mathrm{Cu}$, коэрцитивная сила и поле обменного смещения ведут себя одинаково - зависят друг от друга линейно. Это происходит потому, что обменное взаимодействие одинаково изменяет оба параметра $H_{b}$ и $H_{c}$ с ростом толщины слоя меди. Отклонения от линейности означали бы наличие доменной структуры, дефектов, приводящих к сложной зависимости коэрцитивной силы от толщины немагнитной прослойки. Из рис. 6 следует, что этих эффектов нет, то есть коэрцитивная сила управляется прямо обменным взаимодействием и величиной магнитной анизотропии во всем диапазоне температур и исследуемых толщин прослойки меди. Перемагничивание структур происходит путем когерентного вращения вектора намагниченности.

C помощью моделирования процесса напыления меди можно установить отношение коэффициентов адгезии $\mathrm{Cu}-\mathrm{Cu}$ и $\mathrm{Cu}-\mathrm{NiFe}$. Увеличение толщины немагнитной прослойки $\mathrm{Cu}$ моделировали методом Монте-Карло на площадке $100 \times 100$ атомов размером $20 \times 20 \mathrm{~nm}$. Двумерное распределение кластеров $\mathrm{Cu}$ получали дискретным добавлением случайно распределенных 5000 атомов на каждом шаге, что соответствует половине монослоя (ML), покрывающего площадку. Эксперимент завершали при добавлении 5 атомных монослоев или 50000 атомов. В алгоритме было задано, что вероятность закрепления атома $\mathrm{Cu}$ на непокрытой поверхности $\mathrm{NiFe}$ и на участок, покрытый медью, различна. Это различие вероятностей захвата атома на разные участки задает соответствующее отношение $r$ коэффициента адгезии $\mathrm{Cu}-\mathrm{Cu}$ к коэффициенту адгезии $\mathrm{Cu}-\mathrm{NiFe}$. Таким образом, получали серию зависимостей доли покрытой площади от количества осажденных атомов при разных отношениях $r$ коэффициентов адгезии $\mathrm{Cu}-\mathrm{Cu}$ и $\mathrm{Cu}-\mathrm{NiFe}$ в диапазоне от 1 до $10^{5}$. Критерием для выбора $r$ служило предположение о том, что отношение поля обменного смещения, вызванное медью, к исходному обменному смещению без меди $H_{b}\left(t_{\mathrm{Cu}}\right) / H_{b 0}$ при изменении толщины слоя меди должно следовать отношению площади непокрытой части поверхности $S_{\mathrm{NiFe}}$ к площади покрытой поверхности $S_{\mathrm{Cu}}$. Поэтому среди полученных зависимостей доли покрытой площади от количества осажденных атомов, выбирали ту, для которой выполняется соотношение $H_{b}\left(t_{\mathrm{Cu}}\right) / H_{b 0}=S_{\mathrm{NiFe}} / S_{\mathrm{Cu}}$ при всех толщинах слоя меди $t_{\mathrm{Cu}}$. На рис. 7, $a$ сплошной линией показан такой случай, который соответствует $r(\mathrm{Cu})=2500$. При этом вероятность удержания атома меди на покрытой поверхности была равна 0.25 , а на непокрытой поверхности $\mathrm{NiFe}-0.0001$. На рис. $7, b$ в качестве примера показан результат моделирования распределения кластеров $\mathrm{Cu}$, при эффективной толщине 

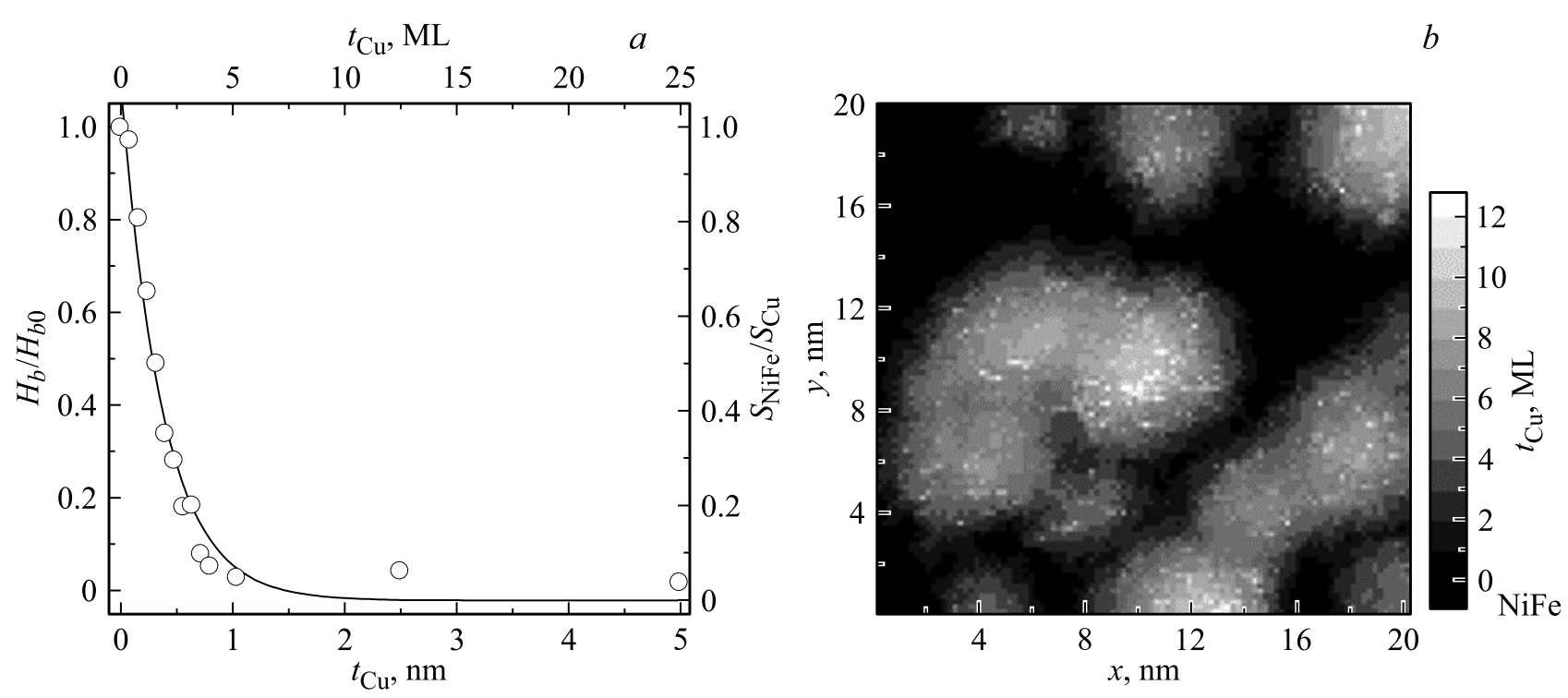

Рис. 7. Зависимость отношения поля обменного смещения к исходному обменному смещению без меди от эффективной толщины немагнитной прослойки $\mathrm{Cu}$ (символы) и зависимость доли покрытой площади от количества осажденных атомов, выраженного в моноатомных слоях ML (сплошная линия), при отношении коэффициентов адгезии $\mathrm{Cu}-\mathrm{Cu} \kappa \mathrm{Cu}-\mathrm{NiFe}, r(\mathrm{Cu})=2500(a)$. Результат моделирования распределения кластеров $\mathrm{Cu}$ с параметрами, описанными в тексте $(b)$.

немагнитной прослойки $t_{\mathrm{Cu}}=0.6 \mathrm{~nm}(3.0 \mathrm{ML})$. Модель предсказывала полное покрытие медью при эффективной толщине слоя $1 \mathrm{~nm}$. В экспериментах при достижении толщины слоя меди $1 \mathrm{~nm}$ возникает резкое изменение коэрцитивной силы и поля обменного смещения гетероструктур (см. вставки к рис. 5 в двойных логарифмических координатах). Это говорит о правильно выбранных коэффициентах адгезии меди и хорошей предсказательной силе модели.

\section{4. Выводы}

1. Увеличение эффективной толщины слоя меди в структурах $\mathrm{NiFe} / \mathrm{Cu} / \mathrm{IrMn}$, происходящее через стадии частичного покрытия, возникновения островков разной толщины и размера, образование бесконечного фрактала, полного покрытия и увеличение толщины сплошной пленки, на всех этих стадиях не меняет спектров рентгеновской дифракции и степень совершенства магнитоупорядоченных слоев и не затрагивает процесс перемагничивания когерентным вращением намагниченности в диапазоне температур $2-300 \mathrm{~K}$.

2. Температурные зависимости обменного смещения и коэрцитивной силы структур описываются суммой двух компонент с разным обменным взаимодействием, соответствующим участкам с прямым контактным обменом $\mathrm{NiFe} / \mathrm{IrMn}$ и участкам с косвенным РККИ обменом $\mathrm{NiFe} / \mathrm{Cu} / \mathrm{IrMn}$.

3. Зависимость коэрцитивной силы и обменного смещения от эффективной толщины слоя меди содержат стадии, указывающие на наличие нескольких промежуточных способов покрытия магнито-упорядоченного материала медью.
4. Моделирование процесса возникновения островков показывает, что при отношении коэффициентов адгезии к меди и к $\mathrm{NiFe}$, равном 2500, сплошное покрытие медью возникает примерно при $1 \mathrm{~nm}$ эффективной толщины слоя меди. Это совпадает с экспериментально определенной толщиной слоя меди $1 \mathrm{~nm}$, при которой возникает резкое изменение коэрцитивной силы и поля обменного смещения гетероструктур.

\section{Финансирование работы}

Работа выполнена при поддержке гранта Президента Российской Федерации для государственной поддержки ведущих научных школ Российской Федерации НШ-2644.2020.2. Работа выполнена в рамках темы AАAА-A19-119111390022-2 госзадания ИПХФ РАН.

\section{Конфликт интересов}

Авторы заявляют, что у них нет конфликта интересов.

\section{Список литературы}

[1] V. Baltz, A. Manchon, M. Tsoi, T. Moriyama, T. Ono, Y. Tserkovnyak. Rev. Mod. Phys. 90, 015005 (2018).

[2] J.Y. Son, C.H. Kim, J.H. Cho, Y.H. Shin, H.M. Jang. ACS Nano 4, 3288 (2010).

[3] R. Stamps. J. Phys. D 33, R247 (2000).

[4] P. Manna, S. Yusuf. Phys. Rep. 535, 61 (2014).

[5] J. McCord, R. Mattheis, D. Elefant. Phys. Rev. B 70, 094420 (2004).

[6] Y.T. Chen. Nanoscale Res. Lett. 4, 90 (2008).

[7] Y. Hu, X. Li, X. Chi, A. Du, F. Shi. J. Phys. D 51, 055001 (2018). 
[8] T.R. Gao, Z. Shi, S.M. Zhou, R. Chantrell, P. Asselin, X.J. Bai, J. Du, Z.Z. Zhang. J. Appl. Phys. 105, 053913 (2009).

[9] N.P. Aley, G. Vallejo-Fernandez, R. Kroeger, B. Lafferty, J. Agnew, Y. Lu, K.O. Grady. IEEE Trans. Magn. 44, 2820 (2008).

[10] H.S. Jung, O. Traistaru, H. Fujiwara. J. Appl. Phys. 95, 6849 (2004).

[11] H. Sang, Y.W. Du, C.L. Chien. J. Appl. Phys. 85, 4931 (1999).

[12] J.P. King, J.N. Chapman, M.F. Gillies, J.C.S. Kools. J. Phys. D 34, 528 (2001).

[13] T.Q. Hung, S. Oh, B. Sinha, J.R. Jeong, D.Y. Kim, C.G. Kim. J. Appl. Phys. 107, 09E715 (2010).

[14] L. Thomas, A.J. Kellock, S.S.P. Parkin. J. Appl. Phys. 87, 5061 (2000).

[15] S. Nicolodi, L.C.C.M. Nagamine, A.D.C. Viegas, J.E. Schmidt, L.G. Pereira, C. Deranlot, F. Petroff, J. Geshev. J. Magn. Magn. Mater. 316, e97 (2007).

[16] J. Sort, F. Garcia, B. Rodmacq, S. Auffret, B. Dieny. J. Magn. Magn. Mater. 272-276, 355 (2004).

[17] K. Li, Z. Guo, G. Han, J. Qiu, Y. Wu. J. Appl. Phys. 93, 6614 (2003).

[18] F. Spizzo, M. Tamisari, F. Chinni, E. Bonfiglioli, L. Del Bianco. J. Magn. Magn. Mater. 421, 234 (2017).

[19] Y.T. Chen, Y.C. Lin, S.U. Jen, J.Y. Tseng, Y.D. Yao. J. Alloys Comp. 509, 5587 (2011).

[20] A. Elzwawy, A. Talantsev, C. Kim. J. Magn. Magn. Mater. 458, 292 (2018).

[21] I.L. Castro, V.P. Nascimento, E.C. Passamani, A.Y. Takeuchi, C. Larica, M. Tafur, F. Pelegrini. J. Appl. Phys. 113, 203903 (2013).

[22] Р.Б. Моргунов, А.Д. Таланцев, М.В. Бахметьев, Н.В. Грановский. ФТТ 6, 915 (2020).

Редактор К.В. Емщев 